Europhysics Letters

PREPRINT

\title{
Bridging the microscopic and the hydrodynamic in active filament solutions
}

\author{
T. B. Liverpool ${ }^{1,2}$ and M. C. Marchetti ${ }^{3}$ \\ 1 Department of Applied Mathematics, University of Leeds, Woodhouse Lane, Leeds LS2 \\ 9JT, $U K$ \\ 2 Isaac Newton Institute for Mathematical Sciences, 20 Clarkson Road, Cambridge CB3 \\ OEH, UK \\ 3 Physics Department, Syracuse University, Syracuse, NY 13244, USA
}

PACS. 87.16.Ka - Filaments, microtubules, their networks, and supramolecular assemblies. PACS. 87.16.Nn - Motor proteins (myosin, kinesin dynein).

\begin{abstract}
Hydrodynamic equations for an isotropic solution of active polar filaments are derived from a microscopic mean-field model of the forces exchanged between motors and filaments. We find that a spatial dependence of the motor stepping rate along the filament is essential to drive bundle formation. A number of differences arise as compared to hydrodynamics derived (earlier) from a mesoscopic model where relative filament velocities were obtained on the basis of symmetry considerations. Due to the anisotropy of filament diffusion, motors are capable of generating net filament motion relative to the solvent. The effect of this new term on the stability of the homogeneous state is investigated.
\end{abstract}

Introduction. - Soft active systems are a new and exciting class of complex fluids to which energy is continuously supplied by internal or external sources. Biology provides many examples of such systems, including cell membranes and biopolymer solutions driven by chemical reactions, living cells moving on a substrate, and the cytoskeleton of eukariotic cells [1]. The cytoskeleton is a complex network of long filamentary proteins (mainly F-actin and microtubules) cross-linked by a variety of smaller proteins $[2,3]$. Among the latter are clusters of motor proteins, such as myosin and kinesin, that use chemical energy from the hydrolysis of ATP to "walk" along the filaments, mediating the exchange of forces between them [4-6].

The self-organization of motor-filament mixtures has been studied by in vitro experiments [4-6]. Complex patterns, including asters and vortices, have been observed as a function of motor and ATP concentration in a confined quasi-two-dimensional geometry $[5,6]$. The high frequency mechanical response of active filament solutions has also been studied both experimentally and theoretically $[7,8]$. The study of these simplified model systems should lead to a better understanding of the formation and stability of more complex structures of biological relevance, such as the mitotic spindle formed during cell division $[1,9]$.

There have been a number of recent theoretical studies of the collective dynamics of rigid active filaments. First and most microscopic, numerical simulations with detailed modelling of the filament-motor coupling have been shown to generate patterns similar to those found

(C) EDP Sciences 
in experiments $[5,6]$. A second interesting development has been the proposal of 'mesoscopic' mean-field kinetic equations where the effect of motors was incorporated via a motor-induced relative velocity of pairs of filaments, with the form of such velocity inferred from general symmetry considerations [10-12]. Finally, hydrodynamic equations have been proposed where the mixture is described in terms of a few coarse-grained fields whose dynamics is also inferred from symmetry considerations [13-19]. Recently, we established a connection between the mesoscopic and hydrodynamic approaches by deriving hydrodynamics via a coarse-graining of the kinetic equations [20]. Both the mesoscopic and hydrodynamic approaches share, however, an important shortcoming. The rate and strength of the motor-induced force exchange among the filaments is controlled by phenomenological parameters whose dependence on motor activity is not known. The richness of the phenomena exhibited by the cytoskeleton leads naturally to the question of how much of the behavior is specific and how much is generic. To answer this question it is important to make the connection between microscopic models and 'generic' hydrodynamic approaches.

In this paper we present a first attempt at deriving the motor-mediated interaction between filaments from a microscopic description of the forces exchanged between the motors and the filaments. Our work establishes the connection between the hydrodynamic equations and the microscopic motor dynamics. In particular, it shows explicitly that in mean-field models of the type considered here spatial inhomogeneities in the motor stepping rate along the filaments are essential to drive bundle formation.

Kinematics of filament pair. - The filaments are modelled as rigid rods of length $l$ (here $l$ should be thought of as the persistence length, rather than the actual filament length) and diameter $b<<l$. Each filament is identified by the location $\mathbf{r}_{i}$ of its center of mass and a unit vector $\hat{\mathbf{n}}_{i}$ pointing towards the polar end. The mobile crosslinks are formed by small aggregates of molecular motors that exert a force on filaments by converting chemical energy from the hydrolysis of ATP into mechanical work. Each motor cluster is assumed to be composed of two heads, with the $i$-th head $(i=1,2)$ attached to filament $i$ at position $\mathbf{r}_{i}^{\times}=\mathbf{r}_{i}+\hat{\mathbf{n}}_{i} s_{i}$, with $s_{i}$ the position along the filament relative to the center of mass, $-l / 2 \leq s_{i} \leq+l / 2$. The motor cluster has size $l_{m}<<l$. Hence the attachment points satisfy $\mathbf{r}_{1}^{\times} \simeq \mathbf{r}_{2}^{\times}$, or $\boldsymbol{\xi}=\mathbf{r}_{2}-\mathbf{r}_{1} \simeq s_{1} \hat{\mathbf{n}}_{1}-s_{2} \hat{\mathbf{n}}_{2}$. A schematic is shown in Fig. 目 Motor heads are assumed to step towards the polar end of filaments at a known speed, $u(s)$, which generally depends on the point of attachment. Both filaments and motors move through a solution. We assume that the filament dynamics is overdamped and the friction of motors is very small compared to that of filaments. Momentum conservation then requires that in the absence of external forces and torques, the total force acting on filaments centered at a given position be balanced by the frictional force experienced by the filament while moving through the fluid.

We consider a pair of filaments cross-linked by a single motor cluster. Due to the action of the motors, filaments 1 and 2 acquire center-of-mass velocities $\mathbf{v}_{1}$ and $\mathbf{v}_{2}$ and rotational velocities $\boldsymbol{\omega}_{1}$ and $\boldsymbol{\omega}_{2}$ about the center of mass. We evaluate these velocities in terms of the known motor stepping rate, $u(s)$, and of the filaments' orientation. In the absence of external forces, any force or torque generated by active crosslinks on one of the filaments of the pair is balanced by an equal and opposite force or torque acting on the other filament. This requires

$$
\begin{aligned}
& \zeta_{i j}\left(\hat{\mathbf{n}}_{1}\right) v_{1 j}=-\zeta_{i j}\left(\hat{\mathbf{n}}_{2}\right) v_{2 j}, \\
& \zeta_{r} \boldsymbol{\omega}_{1}=-\zeta_{r} \boldsymbol{\omega}_{2},
\end{aligned}
$$

where $\zeta_{i j}(\hat{\mathbf{n}})=\zeta_{\|} \hat{n}_{i} \hat{n}_{j}+\zeta_{\perp}\left(\delta_{i j}-\hat{n}_{i} \hat{n}_{j}\right)$ is the friction tensor of the rod, with $\zeta_{\|}$and $\zeta_{\perp}$ the longitudinal and transverse friction coefficients, respectively, and $\zeta_{r}$ is the rotational friction. 


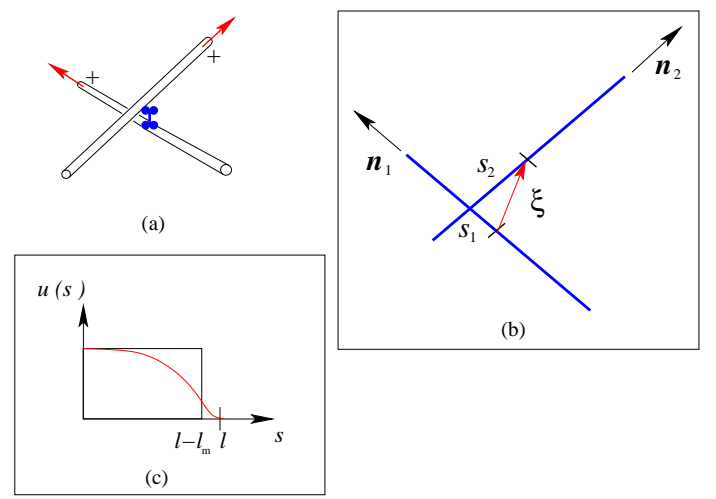

Fig. 1 - (a)Two filaments connected by an active crosslink. (b)The geometry of the overlap: the filaments' centers are separated by $\boldsymbol{\xi}=s_{1} \hat{\mathbf{n}}_{1}-s_{2} \hat{\mathbf{n}}_{2}$. (c) The profile of the motor stepping rate.

The $\alpha$-th motor head $(\alpha=1,2)$ at position $\mathbf{r}_{m \alpha}=\mathbf{r}_{\alpha}+s_{\alpha} \hat{\mathbf{n}}_{\alpha}$ has velocity $\dot{\mathbf{r}}_{m \alpha}=\mathbf{v}_{\alpha}+$ $u_{0}\left(s_{\alpha}\right) \hat{\mathbf{n}}_{\alpha}+s_{\alpha} \boldsymbol{\omega}_{\alpha} \times \hat{\mathbf{n}}_{\alpha}$, where $\dot{s}_{\alpha}=u_{0}\left(s_{\alpha}\right)$ and $\dot{\mathbf{n}}_{\alpha}=\boldsymbol{\omega}_{\alpha} \times \hat{\mathbf{n}}_{\alpha}$. Motors may also rotate relative to the filament at a rate $\boldsymbol{\omega}_{m \alpha}=\boldsymbol{\omega}_{\alpha}+(-1)^{\alpha-1} \dot{\theta}_{\alpha}\left(s_{\alpha}\right)\left(\hat{\mathbf{n}}_{1} \times \hat{\mathbf{n}}_{2}\right) /\left|\hat{\mathbf{n}}_{1} \times \hat{\mathbf{n}}_{2}\right|$, with $\theta_{\alpha}\left(s_{\alpha}\right)$ the angle the $\alpha$-th motor head makes with the filament to which it is attached. If the motor heads are rigidly attached to each other we must have $\dot{\mathbf{r}}_{m 1}=\dot{\mathbf{r}}_{m 2}$ and $\boldsymbol{\omega}_{m 1}=\boldsymbol{\omega}_{m 2}$. Since the motors are assumed to be point-like in size on the scales of interest, we neglect motor rotation below, i.e., $\dot{\theta}_{\alpha}\left(s_{\alpha}\right)=0$, which then requires $\boldsymbol{\omega}_{1}=\boldsymbol{\omega}_{2}=0$. The anisotropy of the rods' friction tensor, allows for both relative and net translation of the filaments induced by the action of the motors. Using Eqs. (1), we find that the relative velocity $\mathbf{v}=\mathbf{v}_{1}-\mathbf{v}_{2}$ of the filaments is

$$
\mathbf{v}=u_{0}\left(s_{2}\right) \hat{\mathbf{n}}_{2}-u_{0}\left(s_{1}\right) \hat{\mathbf{n}}_{1} .
$$

Equations (11) and (3) are readily solved for the filaments velocities, $\mathbf{v}_{1,2}= \pm \mathbf{v} / 2+\mathbf{V}$. The velocity $\mathbf{V}=\left(\mathbf{v}_{1}+\mathbf{v}_{2}\right) / 2$ of the center of mass of the pair is given by

$$
\begin{aligned}
\mathbf{V}= & A\left(\sigma, \hat{\mathbf{n}}_{1} \cdot \hat{\mathbf{n}}_{2}\right)\left\{(1-2 \sigma)\left(\hat{\mathbf{n}}_{1} \cdot \hat{\mathbf{n}}_{2}\right)\left[\hat{\mathbf{n}}_{2} u_{0}\left(s_{1}\right)+\hat{\mathbf{n}}_{1} u_{0}\left(s_{2}\right)\right]\right. \\
& \left.-\left[1-\sigma-\sigma\left(\hat{\mathbf{n}}_{1} \cdot \hat{\mathbf{n}}_{2}\right)^{2}\right]\left[\hat{\mathbf{n}}_{1} u_{0}\left(s_{1}\right)+\hat{\mathbf{n}}_{2} u_{0}\left(s_{2}\right)\right]\right\},
\end{aligned}
$$

where $A=(\sigma / 2)\left[(1-\sigma)^{2}-\sigma^{2}\left(\hat{\mathbf{n}}_{1} \cdot \hat{\mathbf{n}}_{2}\right)^{2}\right]^{-1}$, with $\sigma=\left(\zeta_{\perp}-\zeta_{\|}\right) /\left(2 \zeta_{\perp}\right)$. The fact that $\mathbf{V} \neq 0$ indicates that motor activity can induce a net motion of the pair relative to the solution. This arises from hydrodynamic effects due to the anisotropy of the friction tensor and vanishes when $\zeta_{\perp}=\zeta_{\|}$. Also $\mathbf{V}$ vanishes identically for $\hat{\mathbf{n}}_{2}= \pm \hat{\mathbf{n}}_{1}$, so that $\mathbf{V}=0$ in one dimension.

Derivation of hydrodynamics. - The concentration $c(\mathbf{r}, \hat{\mathbf{n}}, t)$ of filaments with center of mass at $\mathbf{r}$ and orientation $\hat{\mathbf{n}}$ at time $t$ satisfies a local conservation law,

$$
\partial_{t} c=-\nabla \cdot \mathbf{J}-\mathcal{R} \cdot \mathcal{J},
$$

with $\mathcal{R}=\hat{\mathbf{n}} \times \nabla_{\hat{\mathbf{n}}}$. The translational current density, $\mathbf{J}$, and rotational current density, $\mathcal{J}$, are

$$
\begin{aligned}
& J_{i}=-D_{i j} \nabla_{j} c-\frac{D_{i j}}{k_{B} T} c \nabla_{j} V_{\mathrm{ex}}+J_{i}^{\mathrm{act}}, \\
& \mathcal{J}_{i}=-D_{r} \mathcal{R}_{i} c-\frac{D_{r}}{k_{B} T} c \mathcal{R}_{i} V_{\mathrm{ex}}
\end{aligned}
$$


where $D_{i j}=k_{B} T_{a c t} \zeta_{i j}^{-1}=D_{\|} \hat{n}_{i} \hat{n}_{j}+D_{\perp}\left(\delta_{i j}-\hat{n}_{i} \hat{n}_{j}\right)$ is the translational diffusion tensor, with $D_{\|, \perp}=k_{B} T_{a c t} / \zeta_{\|, \perp}$ and $D_{r}=k_{B} T_{a c t} / \zeta_{r}$. The effective active temperature, $T_{a c t}$ may be higher than the ambient temperature $T$ due to the motor activity [8]. The potential $V_{\text {ex }}$ incorporates excluded volume effects which give rise to the nematic-transition in a hard-rod solution. It can be written as $k_{B} T$ times the probability of finding another rod within the interaction area of a given rod, [21]

$$
V_{\mathrm{ex}}\left(\mathbf{r}_{1}, \hat{\mathbf{n}}_{1}\right)=\frac{k_{B} T}{b^{d}} \int d \mathbf{r}_{2} \int d \hat{\mathbf{n}}_{2} \int_{s_{1}} \int_{s_{2}} \delta\left(\mathbf{r}_{1}^{\times}-\mathbf{r}_{2}^{\times}\right) c\left(\mathbf{r}_{2}, \hat{\mathbf{n}}_{2}, t\right) \vartheta\left(\hat{\mathbf{n}}_{1}, \hat{\mathbf{n}}_{2}\right),
$$

where $\int_{s} \ldots \equiv b^{d-1} \int_{-l / 2}^{l / 2} d s \ldots$ and $\vartheta\left(\hat{\mathbf{n}}_{1}, \hat{\mathbf{n}}_{2}\right)=\sqrt{1-\left(\hat{\mathbf{n}}_{1} \cdot \hat{\mathbf{n}}_{2}\right)^{2}}$.

The active current of filaments with center of mass at $\mathbf{r}_{1}$ and orientation along $\hat{\mathbf{n}}_{1}$ is

$$
\begin{aligned}
\mathbf{J}^{\text {act }}\left(\mathbf{r}_{1}, \hat{\mathbf{n}}_{1}\right)=\int d \mathbf{r}_{2} \int d \hat{\mathbf{n}}_{2} \int_{s_{1}} \int_{s_{2}} & \vartheta\left(\hat{\mathbf{n}}_{1}, \hat{\mathbf{n}}_{2}\right) m\left(\mathbf{r}_{1}^{\times}\right) \mathbf{v}_{1}\left(s_{1}, s_{2}, \hat{\mathbf{n}}_{1}, \hat{\mathbf{n}}_{2}\right) \delta\left(\mathbf{r}_{1}^{\times}-\mathbf{r}_{2}^{\times}\right) \\
& \times c\left(\mathbf{r}_{1}, \hat{\mathbf{n}}_{1}, t\right) c\left(\mathbf{r}_{2}, \hat{\mathbf{n}}_{2}, t\right),
\end{aligned}
$$

where $m(\mathbf{r})$ is the density of motor clusters, evaluated at the point of attachment. Finally, $\mathbf{v}_{1}\left(s_{1}, s_{2}, \hat{\mathbf{n}}_{1}, \hat{\mathbf{n}}_{2}\right)$ is the velocity that filament 1 acquires at the point of attachment of the motor cluster due to interaction with filament 2 , when the centers of mass of the two filaments are separated by $\boldsymbol{\xi}=\mathbf{r}_{2}-\mathbf{r}_{1} \simeq \hat{\mathbf{n}}_{1} s_{1}-\hat{\mathbf{n}}_{2} s_{2}$, as obtained earlier. Using the $\delta$-function to carry out the integration over $\mathbf{r}_{2}$, and assuming a uniform density of motor clusters $m_{0}$, we obtain

$$
\begin{aligned}
\mathbf{J}^{\mathrm{act}}\left(\mathbf{r}_{1}, \hat{\mathbf{n}}_{1}\right)=m_{0} \int d \hat{\mathbf{n}}_{2} \int_{s_{1}} \int_{s_{2}} \vartheta\left(\hat{\mathbf{n}}_{1}, \hat{\mathbf{n}}_{2}\right) & \mathbf{v}_{1}\left(s_{1}, s_{2}, \hat{\mathbf{n}}_{1}, \hat{\mathbf{n}}_{2}\right) c\left(\mathbf{r}_{1}, \hat{\mathbf{n}}_{1}, t\right) \\
& \times c\left(\mathbf{r}_{1}+\hat{\mathbf{n}}_{1} s_{1}-\hat{\mathbf{n}}_{2} s_{2}, \hat{\mathbf{n}}_{2}, t\right) .
\end{aligned}
$$

The effect of fluctuations in $m(\mathbf{r})$ will be discussed elsewhere.

To describe filament dynamics on scales large compared to their length $l$, we expand the concentration of filaments $c\left(\mathbf{r}_{1}+\boldsymbol{\xi}, \hat{\mathbf{n}}_{2}\right)$ near its value at $\mathbf{r}_{1}$. After inserting this expansion in Eq. (10), the integrals over $s_{1}$ and $s_{2}$ can be carried out term by term and we find

$$
\begin{aligned}
J_{i}^{\text {act }}\left(\mathbf{r}_{1}, \hat{\mathbf{n}}_{1}\right)=m_{0} \int d \hat{\mathbf{n}}_{2} \quad & c\left(\mathbf{r}_{1}, \hat{\mathbf{n}}_{1}, t\right)\left\{\left\langle v_{1 i}\right\rangle_{s_{1}, s_{2}}+\left\langle v_{1 i} \xi_{j}\right\rangle_{s_{1}, s_{2}} \nabla_{1 j}\right. \\
& \left.+\frac{1}{2}\left\langle v_{1 i} \xi_{j} \xi_{k}\right\rangle_{s_{1}, s_{2}} \nabla_{1 j} \nabla_{1 k}+\ldots\right\} c\left(\mathbf{r}_{1}, \hat{\mathbf{n}}_{2}, t\right),
\end{aligned}
$$

where $\left\langle v_{1 i} \xi_{j} \xi_{k} \ldots\right\rangle_{s_{1}, s_{2}}=\int_{s_{1}} \int_{s_{2}} \vartheta v_{1 i} \xi_{j} \xi_{k} \ldots$ are moments of the local filament velocity. These are expressed in terms of moments of the stepping speed, $u^{(n)}=\int_{-l / 2}^{l / 2}(d s / l)(s / l)^{n} u(s)$. If $u(s)$ is constant, all odd moments, which control the bundling instability of the homogeneous state discussed earlier $[11,20]$, vanish. In mean-field models of the type considered here an inhomogeneous velocity profile is crucial to obtain bundling. Spatial variations of $u(s)$ may for instance arise from motors slowing down as they approach the polar end of the filament due to crowding. This is incorporated here by using the step-like speed profile shown in Fig. [1 where $u(s)$ is constant along the filament, but vanishes in a small region of extent $l_{m}<<l$ at the polar end. Using this form, we estimate $u^{(2 n)} \sim u_{0}$ and $u^{(2 n+1)} \sim-u_{0}\left(l_{m} / l\right)$. All odd moments are negative as filaments slow down as they approach the polar end.

As in Ref. [20] we now assume that on large scales the system dynamics can be described in terms of a local filament density $\rho(\mathbf{r}, t)$ and a local filament polarization, $\mathbf{p}(\mathbf{r}, t)$, defined as 
the first two moments of the distribution $c(\mathbf{r}, \hat{\mathbf{n}}, t)$,

$$
\begin{aligned}
& \rho(\mathbf{r}, t)=\int_{\hat{\mathbf{n}}} c(\mathbf{r}, \hat{\mathbf{n}}, t), \\
& \mathbf{p}(\mathbf{r}, t)=\int_{\hat{\mathbf{n}}} \hat{\mathbf{n}} c(\mathbf{r}, \hat{\mathbf{n}}, t) .
\end{aligned}
$$

Hydrodynamic equations for these coarse-grained densities can then be obtained by writing an exact moment expansion for $c(\mathbf{r}, \hat{\mathbf{n}}, t)$ and truncating it at the second moment. The integrals over the unit vectors $\hat{\mathbf{n}}_{1}$ and $\hat{\mathbf{n}}_{2}$ are carried out by approximating the prefactor $A$ of Eq. (4) and the excluded volume term $\vartheta$ with their mean value over the range of integration. This approximation does not affect the structure of the hydrodynamic equations, but only the numerical values of the coefficients.

The nonlinear hydrodynamic equations for the filament density and local orientation of a mixture of arbitrarily-shaped filaments and motors in $d$ dimensions will be given elsewhere. Here we restrict ourselves to the specific case of long thin rods in $d=2$, where $D_{\|}=2 D_{\perp} \equiv D$. We also linearize the equations around the homogeneous isotropic state, by letting $\rho=\rho_{0}+\delta \rho$ and $\mathbf{p}=\delta \mathbf{p}$. The linearized equations in $d=2$ are

$$
\begin{aligned}
\partial_{t} \delta \rho= & \frac{3}{4} D \nabla^{2} \delta \rho-\frac{\alpha \tilde{\rho}_{0}}{2} \nabla^{2} \delta \rho+\frac{\beta \tilde{\rho}_{0}}{4 \sqrt{2}} \boldsymbol{\nabla} \cdot \mathbf{p} \\
& -\frac{5 \beta \tilde{\rho}_{0} l^{2}}{192}\left(1-\frac{5}{24 \sqrt{2}}\right) \nabla^{2} \boldsymbol{\nabla} \cdot \mathbf{p}-\frac{5 \alpha \tilde{\rho}_{0} l^{2}}{384} \nabla^{2} \nabla^{2} \delta \rho, \\
\partial_{t} p_{i}=\quad- & D_{r} p_{i}+\frac{5}{8} D \nabla^{2} p_{i}+\frac{D}{4} \nabla_{i} \boldsymbol{\nabla} \cdot \mathbf{p}+\frac{\beta \tilde{\rho}_{0}}{2}\left(1+\frac{1}{4 \sqrt{2}}\right) \nabla_{i} \delta \rho \\
+ & \frac{5 \beta \tilde{\rho}_{0} l^{2}}{384}\left(1-\frac{5}{24 \sqrt{2}}\right) \nabla_{i} \nabla^{2} \delta \rho,
\end{aligned}
$$

where $\tilde{\rho}_{0}=\rho_{0} v_{0}$ and

$$
\begin{aligned}
& \alpha=-m_{0} v_{0} u^{(1)} \approx m_{0} v_{0} u_{0} l_{m} / \pi \\
& \beta=m_{0} v_{0} u^{(0)}=2 m_{0} v_{0} u_{0} / \pi .
\end{aligned}
$$

The parameter $\alpha$ has the dimensions of a diffusion constant and describes filament bunching or bundling, which, in contrast to conventional diffusion, tends to enhance density fluctuations. The coefficient $\beta$ is a velocity and describes the rate at which motor clusters sort or separate filaments of opposite polarity. If the motor stepping speed $u(s)$ is constant, independent of the position $s$ along the filament, then $\alpha=0$. In general, even when $\alpha \neq 0$, we expect $\alpha<<\beta$.

The hydrodynamic equations obtained here by using a microscopic model for the motorinduced filament velocities differ from those derived in Ref. [20] where these velocities where written down on the basis of symmetry in two important ways:

1. The density equation contains a new term $\sim \beta \boldsymbol{\nabla} \cdot(\rho \mathbf{p})$ that describes filament convection along the direction of local alignment. This term vanishes for isotropic objects with $\zeta_{\perp}=\zeta_{\|}$. It arises for rods because in this case the motors can generate net motion of the filaments relative to the solution $(\mathbf{V} \neq 0)$.

2. The second important difference is that there are no $\alpha$-type contributions to the active currents proportional to gradients of the local polarization. These leads to the absence of 
EUROPHYSICS LETTERS

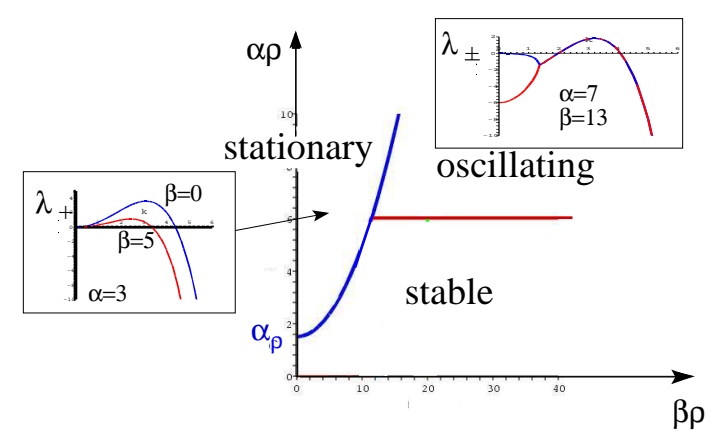

Fig. 2 - The phase diagram in the $\tilde{\alpha}-\tilde{\beta}$ plane showing the different regimes calculated from a low $k$ expansion of $\lambda_{+}$and the eigen-vector $e_{+}^{L, R}$ for $\tilde{\rho}_{0}=0.9$. The insets show the unstable modes.

terms of the form $\sim \nabla_{i}\left[p_{j} \nabla_{j} p_{i}+\ldots\right]$ in the density equation and of terms $\sim \nabla_{j} \rho\left[\nabla_{j} p_{i}+\ldots\right]$ in the polarization equation. These terms are allowed by symmetry and are indeed present in hydrodynamic models written down on the basis of symmetry consideration. $[11,20]$ They vanish, however, upon coarse-graining, when averaging over the length of the filaments. The absence of such terms does not change the bundling instability of density fluctuations, but it does eliminate the possibility of the orientational instability of polarization fluctuations observed in [20] at high filament concentrations.

The absence of terms proportional to polarization gradients in the active current is not related to the fact that our model does not incorporate any motor-induced filament rotation. It arises instead from the fact that the motor dynamics is described in terms of the rate $u(s)$ at which each motor head steps on each filament. These terms then average out when the force exchanged between the two filaments of a pair is averaged along the length of each filament.

Stability Analysis. - To determine the linear stability of the isotropic, homogeneous state, we expand density and polarization fluctuations in Fourier modes, $\delta \rho(\mathbf{r})=\int_{k} e^{i \mathbf{k} \cdot \mathbf{r}} \rho_{k}, \mathbf{p}(\mathbf{r})=$ $\int_{k} e^{i \mathbf{k} \cdot \mathbf{r}} \mathbf{p}_{k}$. Defining the longitudinal polarization mode $p_{k}^{L}=\hat{\mathbf{k}} \cdot \mathbf{p}_{k}$ and $\vec{V}=\left(\delta \rho, p_{k}^{L}\right)$, Eqns. (1314) are equivalent to a matrix equation $\partial_{t} \vec{V}=\mathbb{M} \cdot \vec{V}$. The instability of the system to density and longitudinal polarization fluctuations is determined the range of parameters where for some range of $\mathbf{k}$-vectors, the largest of the eigenvalues $\lambda_{ \pm}(\mathbf{k})$ of the matrix $\mathbb{M}\left(k, \tilde{\rho}_{0}, \tilde{\alpha}, \tilde{\beta}\right)$ has a positive real part. Expressing lengths in units of $l$ and time in units of $l^{2} / D$ we define two dimensionless parameters $\tilde{\alpha}=\alpha / D$ and $\tilde{\beta}=l \beta / D[22]$. For $\tilde{\beta}=0$ the equations are decoupled. In this case there is a stationary instability of density fluctuations for $\tilde{\alpha}>\tilde{\alpha}_{\rho}^{0}=3 /\left(2 \tilde{\rho}_{0}\right)$ and $0<k<k_{\alpha}^{0}$, with $k_{\alpha}^{0} \sim\left(\tilde{\alpha}-\tilde{\alpha}_{c}^{0}\right)^{1 / 2}$. A finite, but small value of the sorting rate $\tilde{\beta}$ tends to stabilize the homogeneous state. This is easily seen from the linearized equations as for times $\gg D_{r}^{-1}$ longitudinal polarization fluctuations simply follow density fluctuations, and to leading order in the gradients $p_{k}^{L} \sim\left[\beta \tilde{\rho}_{0} /\left(2 D_{r}\right)\right] i k \rho_{k}$. Eliminating the longitudinal polarization in the equation for the density, we find that a finite sorting rate $\tilde{\beta}$ effectively enhances diffusion, shifting the instability to $\tilde{\alpha}_{\rho}(\tilde{\beta})=3 /\left(2 \tilde{\rho}_{0}\right)+\left(\tilde{\beta}^{2} \tilde{\rho}_{0}^{2}(1+1 /(4 \sqrt{2})) /(24 \sqrt{2})\right.$. The range of wavevectors over which the modes are unstable is again $0 \leq k \leq k_{\alpha}(\tilde{\beta})$, with $k_{\alpha}(\tilde{\beta}) \sim\left[\tilde{\alpha}-\tilde{\alpha}_{\rho}(\tilde{\beta})\right]^{1 / 2}$. For larger values of $\tilde{\beta}$ the modes are complex conjugate, describing propagating fluctuations. The homogeneous state becomes unstable via an oscillatory instability. The location of the instability is still, however, controlled solely by the bundling coefficient $\tilde{\alpha}$. It occurs at the $\tilde{\beta}$-independent value, $\tilde{\alpha}_{m} \approx 7.6 / \tilde{\rho}_{0}$ above which $\operatorname{Re}\left(\lambda_{ \pm}(k)\right)$ has 
a positive maximum, for an intermediate range of wavenumbers. The actual boundaries of the various regions have been evaluated numerically and are shown in Fig. 2. To establish which order parameter (density, polarization, or both) goes unstable, we have evaluated the right and left eigen-vectors associated with the unstable mode, defined by $\mathbb{M} \cdot \vec{e}_{ \pm}^{R}(k)=\lambda_{ \pm}(k) \vec{e}_{ \pm}^{R}(k)$ and $\vec{e}_{ \pm}^{L}(k) \cdot \mathbb{M}=\lambda_{ \pm}(k) \vec{e}_{ \pm}^{L}(k)$, respectively. The unstable eigen-vector associated with the stationary instability at $\tilde{\alpha}_{\rho}(\tilde{\beta})$ is dominated by density fluctuations at all length scales. The unstable eigen-vector associated with the oscillatory instability at $\tilde{\alpha}_{m}$ is always a mixed one which crosses over from density at small $k$ to longitudinal polarization at large $k$.

In summary, the homogeneous state is always driven unstable by a sufficiently large value of the bundling constant $\alpha$. The polarization sorting rate $\beta$ tends to stabilize the homogeneous state. A large $\beta$ also changes the nature of the instability from stationary to oscillatory. Finally, an important consequence of the absence of $\sim \alpha$-type contributions to the active currents proportional to polarization gradients is that our model yields no pure polarization instability at large length scales. In particular, fluctuations in the transverse part of the polarization $(\sim \nabla \times \mathbf{p})$ that may control the onset of vortex-type structures, are always stable to linear order. A numerical solution of the full nonlinear equations is, however, needed to establish the precise nature of the inhomogeneous states.

TBL acknowledges the support of the Royal Society. MCM acknowledges support from the National Science Foundation, grant DMR-0305407.

\section{REFERENCES}

[1] B. Alberts et AL, Molecular Biology of the Cell (Garland, New York) 2002.

[2] J. Howard, Mechanics of Motor Proteins and the Cytoskeleton (Sinauer, New York) 2000.

[3] M. Dogterom, A. C. Maggs and S. Leibler, Proc. Nat. Acad. Sci. USA, 92 (1995) 6683.

[4] K. Takiguchi, J. Biochem., 109 (1991) 520; R. Urrutia et al., PNAS, 88 (1991) 6701.

[5] F. J. Nédélec, T. Surrey, A. C. Maggs and S. Leibler, Nature, 389 (1997) 305.

[6] T. Surrey, F. J. Nédélec, S. Leibler and E. Karsenti, Science, 292 (2001) 1167.

[7] D. Humphrey, C. Duggan, D. Saha, D. Smith and J. Käs, Nature, 416 (2002) 413.

[8] T. B. Liverpool, A.C. Maggs and A. Ajdari, Phys. Rev. Lett., 86 (2001) 4171.

[9] E. Cytrynbaum, V. Rodionov and A. Mogilner, J. Cell Sci., 117 (2004) 1381.

[10] H. Nakazawa and K. Sekimoto, J. Phys. Soc. Jpn., 65 (1996) 2404; K. Sekimoto And H. Nakazawa, in Current Topics in Physics, edited by Y. M. Cho, J B. Homg and C. N. Yang (World Scientific, Singapore)1998.

[11] K. Kruse and F Jülicher, Phys. Rev. Lett., 85 (2000) 1778; Phys. Rev. E, 67 (2003) 051913.

[12] K. Kruse, S. Camalet and F. Jülicher, Phys. Rev. Lett., 87 (2001) 138101.

[13] H. Y. Lee and M. Kardar, Phys. Rev. E, 64 (2001) 56113.

[14] J. Kim et Al., J. Korean Phys. Soc., 42 (2003) 162.

[15] S. Sankararaman, G. I. Menon and P.B. S. Kumar, arXiv:cond-mat/0307720

[16] K. Kruse et al, Phys. Rev. Lett., 92 (2004) 078101.

[17] R. Aditi Simha and S. Ramaswamy, Phys. Rev. Lett., 89 (2002) 058101.

[18] Y. Hatwalne et Al., Phys. Rev. Lett., 92 (2004) 118101.

[19] B. Bassetti, M. C. Lagomarsino and P. Jona, Eur. Phys. J. B, 15 (2000) 483.

[20] T. B. Liverpool and M. C. Marchetti, Phys. Rev. Lett., 90 (2002) 138102.

[21] M. Doi and S. F. Edwards, The theory of polymer dynamics (Oxford University Press, New York) 1986.

[22] For the specific form of $u(s)$ used here, $\tilde{\alpha}=\tilde{\beta}\left(l_{m} / l\right)$. 